\title{
Análisis Cualitativo de la Personalidad de una Actriz. Estudio de Caso y Trastorno de Personalidad Límite
}

\section{A Qualitative Analysis of the Personality of an Actress. Case Study and Borderline Personality Disorder}

\author{
Margarita Ortiz-Tallo y Marta Ferragut \\ Universidad de Málaga
}

\begin{abstract}
Resumen. Se analiza la historia de la vida de una famosa actriz actualmente fallecida. Los datos se recogen a través del estudio y lectura de documentos y biografías. Se realiza, por tanto, un estudio de caso a través de documentos publicados en una investigación retrospectiva. Se concluye la posible existencia de un Trastorno Reactivo de la Vinculación en la infancia y de un Trastorno de Personalidad Límite en la vida adulta y se justifica. Por último, se explica y profundiza en las características del Trastorno de Personalidad Límite y se mencionan hipótesis explicativas de este trastorno.

Palabras clave: personalidad límite, estudio de caso, trastornos de personalidad, trastorno reactivo de la vinculación en la infancia.
\end{abstract}

\begin{abstract}
This paper analyzes the life story of a famous actress now deceased. Data are collected through studies and reading of documents and biographies. A retrospective case study through documents is presented. We conclude the possible existence of a Reactive Attachment Disorder in childhood and a Borderline Personality Disorder in adulthood and is justified. Finally, characteristics of borderline personality disorder are explained and hypothesis related disorder are commented.

Key words: borderline personality, case study, personality disorder, reactive attachment disorder in childhood.
\end{abstract}

El objetivo de este trabajo es acercar al lector a la comprensión de un trastorno de Personalidad complejo y grave, como es el trastorno de personalidad límite. En el caso que aquí se plantea se hace también posible el acercamiento al diagnóstico de un trastorno en la Infancia, igualmente complejo y además infrecuente, como es el Trastorno Reactivo de la Vinculación. La intención es colaborar así en la comprensión, a través de la historia de vida de un personaje famoso, de los comportamientos y la

La correspondencia sobre este artículo puede dirigirse a la primera autora al E.mail:mortiz@uma.es forma de afrontamiento de las personas con este tipo de dificultades.

El escoger un personaje famoso puede permitir hacerse una idea más clara del aspecto y del tipo de alteración estudiada, acercándonos de una manera más real al personaje. Asimismo, permite exponer un ejemplo, sin faltar a la confidencialidad. Al basar la historia en datos publicados por biógrafos de un personaje del que mucho se ha hablado y discutido, no se desvela a lo largo de esta exposición ningún aspecto de su vida que no haya sido publicado y al que cualquier persona pueda acceder, respetando de 
esta forma los aspectos relacionados con el secreto profesional.

Al realizar el estudio del caso sin la presencia de la protagonista y basándonos en documentos públicos, parece de interés justificar brevemente el modo en el que se recogen los datos.

Los datos que se expondrán han sido recopilados a través de la lectura de documentos. En concreto diversas biografías realizadas por distintos autores sobre un personaje famoso del cine (Guiles, 1985; Leaming, 2007; Pepitone y Stadiem, 1979; Reig, 2005; Schneider, 2008; Spoto, 2000). El modo de recoger la información sitúa el estudio del caso en una investigación retrospectiva con las limitaciones que ello supone. Las autoras del estudio, por tanto, no han entrevistado directa y personalmente al personaje ni a ninguno de los informantes, sino que las entrevistas y los datos han sido recopilados por diversos biógrafos y analizados posteriormente.

Los biógrafos se han apoyado para realizar su historia de vida en numerosas entrevistas con personas que conocieron y convivieron con la protagonista. Así se han recogido recuerdos y experiencias de sus distintos maridos, de una hermanastra, de sus agentes publicitarios y de amigas de diferentes épocas de su vida. También se han basado en comentarios públicos y privados, realizados por la protagonista a lo largo de sus últimos años. Toda la información así recogida conforman los elementos claves para la comprensión del caso.

\section{Exposición del caso}

\section{Datos personales}

Se trata de una mujer, casada en cuatro ocasiones, sin hijos y de profesión actriz. Nacida en Junio de 1926 y fallecida, a los 36 años el 5 de Agosto de 1962.

\section{Antecedentes Familiares}

Comenzamos haciendo una breve descripción de su madre para situarnos en el momento del nacimiento. Gladys, la madre de la protagonista, se casa muy joven y tiene dos hijos con su primer marido que enseguida la abandona, y al no ser ella capaz de cuidar a sus hijos los envía con unos parientes a otra ciudad. Es descrita como una mujer de carácter inestable, inseguro y frágil, así como con una enorme capacidad de seducción apasionándole el mundo de las estrellas y el glamour que rodea a los artistas.

Tiene varios amantes, vuelve a contraer matrimonio y en uno de estos periodos se queda de nuevo embarazada sin saber con certeza quien es el padre de la nueva criatura. Así, en este ambiente de inestabilidad nace la protagonista de nuestra historia, a la que ponen el nombre de Norma J. Pocos días después de su nacimiento la niña es dejada por su madre al cuidado de una familia adoptiva que vive a veinticinco kilómetros de distancia y que se dedica a cuidar bebés cobrando por ello. Así se iniciará el difícil peregrinar de la protagonista en busca de un hogar, búsqueda infructuosa a lo largo de su vida.

\section{Infancia}

La primera infancia de la protagonista es bastante estable geográficamente, ya que sus primeros siete años de vida los pasa en el modesto apartamento de sus cuidadores adoptivos.

Durante esa época, su abuela materna, de nombre Della, que había pasado por períodos de internamiento en un sanatorio mental y por períodos de mayor estabilidad en los que visitaba a su nieta, fallece, figurando en su certificado de defunción como causa, una miocarditis, a la que contribuyó una psicosis maniaco-depresiva. Este diagnóstico psicopatológico añadido, probablemente es poco preciso, no sólo por la época en la que nos situamos, sino que además es anotado al insistir sus parientes en que las últimas semanas el humor y el carácter cambiaban de manera imprevisible.

Desde el punto de vista de los biógrafos, Della, la abuela, muere de una enfermedad cardiaca que le causa un deterioro mental como consecuencia de una insuficiente oxigenación del cerebro. Llegando a la conclusión, que al igual que en el caso de su primer marido, ingresado también en un sanatorio mental, no hay pruebas suficientes que confirmen que son casos psiquiátricos. Sin embargo, para 
Gladys, madre de la protagonista y para ésta última, el mito de la locura familiar se acentúa quedando para siempre el temor de pertenecer a una familia con alteraciones psiquiátricas que podían seguir heredando.

Durante estos siete primeros años de su vida Norma, parece ser que contradiciendo informaciones posteriores emitidas por intereses publicitarios, vive una época en la que no tiene necesidades materiales, de la que no existen pruebas de que recibiera malos tratos, pero sin embargo, marcada por la tensión psicológica y emocional de su dudosa identidad, y de saber que su madre biológica puede aparecer repentinamente y desparecer con igual rapidez.

Destacar de este periodo de su formación la educación impartida por sus padres adoptivos, que la protagonista definiría posteriormente como "terriblemente estricta”. Así como una relación desprovista de elogios o comentarios agradables. En este sentido, Norma diría "en esa casa nadie me dijo jamás que era bonita".

La familia da prioridad en la educación a la moralidad y a las responsabilidades piadosas, siendo la iglesia el centro de su vida y por extensión la de todos los niños a su cuidado. Una de las oraciones repetidas cada noche incluía frases como: "Prometo con la ayuda de Dios no comprar, beber, vender ni dar alcohol mientras viva. Me abstendré de probar el tabaco y nunca tomaré el nombre de Dios en vano". También el cine y el mundo de los actores es considerado pecaminoso.

La vida en estos años es tan previsible y organizada, que Norma recuerda posteriormente que se aficiona a inventarse historias para salir de esa monotonía.

Inmediatamente después de cumplir los siete años, su vida cambia radicalmente al decidir su madre, Gladys, llevársela a vivir con ella. Gladys comparte casa y gastos con actores de poca fortuna y entre todos presentan un ambiente a la pequeña diametralmente opuesto al que conocía en sus primeros años de vida. La vida en general, para los adultos con los que convive es despreocupada y tumultuosa, bailan, beben y la casa está abierta a todo tipo de visitas.

La marcada diferencia entre las nuevas actitudes educativas y las de su primera infancia causan en la pequeña una considerable confusión acerca de la conducta adecuada y de las pautas de lo correcto y de lo incorrecto, comentando en su época adulta las siguientes palabras: "En esa época me sentía un poco escandalizada, pensaba que todos irían al infierno y me pasaba horas rezando por todos ellos".

Al cumplir la niña ocho años, su bisabuelo materno al que ni ella ni su madre habían conocido, se suicida colgándose de una viga. Esta noticia hace que su madre, Gladys, asustada de la maldición familiar y convencida de que existía una plaga de enfermedades mentales en su familia, entre en un estado de estupefacción y depresión en el que comienza a ser medicada.

Desde el punto de vista de los biógrafos, el tratamiento psiquiátrico que recibe Gladys en esos momentos es inadecuado, en gran parte, por ser la psiquiatría un campo médico, que se encontraba en los años treinta en sus inicios. A partir de estos momentos comienza un periodo de ingresos periódicos en un centro psiquiátrico que dejan de nuevo a la niña sin su madre.

Comienza a ser cuidada por una íntima amiga de su madre, Grace, que estimula a la niña hacia nuevas pautas de conducta en la que la seducción, la imitación de artistas y la vestimenta llamativa y no muy adecuada para su edad marcan este nuevo periodo de su vida. Quedando así definida su infancia por una secuencia continua de contradicciones.

A los nueve años Grace, al no poder ocuparse de la niña, la ingresa en un orfelinato, en el que estaría por un período de dos años hasta cumplir los 11, aunque siendo visitada por esta tercera figura materna, que quiere hacer de la niña una futura actriz.

La educación del orfelinato parece haber sido menos sectaria. Los supervisores alientan a los niños a asistir a la iglesia pero de una forma mucho menos rígida y en la ficha del Colegio, Norma queda descrita como "una niña normal, sana que come y duerme bien, que parece contenta, no se queja y dice que le gustan sus clases".

A los 11 años, sin embargo, el temperamento de la niña se vuelve más sombrío y se aconseja que salga del Colegio.

Grace, la amiga de su madre, que durante ese tiempo ha contraído matrimonio, la adopta formalmente y se la lleva a vivir con su esposo y una hija 
de la misma edad que él ha aportado al matrimonio.

Pronto, un episodio desagradable hace que se separen. El marido, una noche que estaba bebido intenta abusar sexualmente de la niña. Esto hace que la envíen a vivir a casa de unos parientes de Grace cambiando de familia en dos ocasiones más hasta cumplir los 13 años. En uno de estos periodos parece que también hay abusos deshonestos por parte de uno de los hijos mayores de la familia de acogimiento.

\section{Adolescencia}

Físicamente se desarrolla antes que la mayoría de las niñas de su edad, siendo cada menstruación durante la mayor parte de su vida unos días penosos y dolorosos que la llevan a recluirse.

Pero también el desarrollo físico le permite destacar entre los chicos y desarrollar una enorme capacidad para seducir y atraer que utiliza constantemente a lo largo de su vida.

Vuelve a vivir con Grace, su marido y la hija de éste que se convierte en una gran amiga. Sin embargo, Norma llora con mucha frecuencia, es desigual en sus reacciones y le hace confidencias a su amiga sobre su sentimiento de vacío y de falta de sentido en su vida.

Al cumplir los 15 años, la familia será destinada a otra ciudad y ante el temor de que prescindan de nuevo de ella y tener que ingresar en un orfelinato decide casarse con un chico mayor que ella que en ese momento la corteja.

\section{Época Adulta}

La vida de la joven esposa atractiva, con un marido que se alista en el ejército, hace que comience una época de continuos amantes que la van llevando en brazos de unos a otros; pero al mismo tiempo cayendo en un estado depresivo cada vez que el esposo después de un permiso, tiene que volver a marcharse. El temor a ser abandonada por su marido y la necesidad de atraer a todo el mundo la hacen entrar en situaciones difíciles así como en períodos de gran abatimiento.
Comienza a consumir drogas tranquilizantes de las que será adicta hasta su muerte. Las personas que la conocen en esta época cuentan que relataba historias melodramáticas sobre su corta pero intensa vida, que vistas por los biógrafos parecen ser retazos de historias de personas que ella ha conocido, entremezcladas con experiencias suyas y que la hacen dibujar un pasado aún más escabroso del que realmente ha tenido.

Durante las ausencias de su marido, se presenta a desfiles de modelo y comienza pequeños papeles como actriz. Durante esa época se sucede el que sería su primer divorcio.

A los 20 años Norma logra su sueño dorado y consigue una cita con el jefe de reparto de la productora de cine FOX. De su primera prueba ya dirían "Además de irradiar sexo en cada fotograma de la prueba, demostró que era capaz de vender emociones. Conseguía transmitir lo que quería sólo con sus expresiones". Sin embargo, sus primeras películas no tienen éxito.

Seguiría unos años más cambiando de productoras, mientras se van sucediendo relaciones amorosas intensas e inestables con productores y actores. Por entonces deciden cambiarle el nombre y ponerle uno más comercial, el de Marilyn Monroe.

La productora le impone una profesora de drama, Natascha, mujer compleja y posesiva, en la que Norma halla una figura de quién depender y con quien se cree mantendrá una relación lésbica. Posteriormente aparecerán otros personajes en su vida, todos ellos femeninos, con los que tendrá relaciones de dependencia extrañas y en las que ella se moverá entre la necesidad de dependencia y la necesidad de rebelarse.

Sus amoríos con distintos hombres también continúan, sucediéndose a lo largo de su vida tres matrimonios más, uno de los cuales sólo llega a durar unos días.

Se podría situar el año 1949, como momento crítico en su ascenso al estrellato, año en el que aparecen unas fotos suyas en el primer número de la revista Play Boy y que harán de Marilyn piedra de escándalo y oscuro objeto de deseo para varias generaciones.

A partir de este momento, comienza una etapa en su carrera en la que directores y productores quedan 
impresionados no sólo de su belleza sino de la enorme facilidad para expresar emociones y sentimientos y de su gran talento para la actuación. Su capacidad de seducción consigue también atraer a un público que pronto la convertiría en mito.

Sin embargo, sus compañeros de reparto se quejan y se desesperan al tener que trabajar con Marilyn. Llega tarde a los rodajes y durante días se encierra en su habitación sin querer ver a nadie. Esto hace que los ensayos, se tengan que suspender y reanudar en función de su estado de ánimo. Saca de quicio a los directores que la describen como una mujer de reacciones caprichosas. Marilyn, a pesar de estar consiguiendo su sueño adolescente, se queja continuamente de su infelicidad.

Durante su época adulta se le conocen variados intentos de suicidio, intenta tirarse por una ventana y toma excesivas pastillas. En varias ocasiones tiene que ser ingresada por ello. Los motivos que la llevan a estos intentos son diversos, algo intrascendente para otros o una pequeña pelea puede hacerla sentir tan mal que intente suicidarse. En otras ocasiones, el temor al abandono del marido del momento o en otro momento, la pérdida de un embarazo, son los desencadenantes de sus comportamientos autolesivos.

Para los que la rodean su absoluta falta de responsabilidad y de profesionalidad está llegando demasiado lejos y es frecuente la paralización de los rodajes de películas debida a sus periodos de crisis. Algunos actores dicen odiarla y no soportan su falta de disciplina y sus continuos caprichos. Pero también pueden encontrarse de un día para otro a una mujer agradable y seductora.

En una de estas épocas cae en una profunda depresión, dice estar cansada de luchar y encontrarse con un desenfrenado sentimiento de autodestrucción que no puede controlar.

Uno de sus maridos dirá posteriormente: "La intensidad de su angustia la habría incapacitado para cualquier otro trabajo. Si hubiese sido oficinista, le habrían dicho que hiciera el favor de quedarse en su casa. Sin embargo, en la vida le tocó el papel de estrella de cine y esto le permitió trabajar y padecer, porque a una estrella se le permite todo".

En diversos rodajes parece confundir realidad y ficción de sus personajes enamorándose locamente de los actores con los que comparte el protagonismo de las películas. En ocasiones para proseguir el rodaje tienen que maquillarla acostada y completamente inerte.

En el año 1961 entra en otro periodo de crisis mayor y es ingresada en un sanatorio mental, cualquier complicación aparentemente sin importancia le hace perder los estribos, sufriendo lo que los médicos denominan "ataques de histeria" que le hacen tirar los muebles de la habitación y proferir gritos a todo el que le rodea.

El médico psiquiatra que la trata durante el último año de su vida encuentra que toma drogas diariamente y extrañado ante la diversidad de síntomas que presenta, realiza un diagnóstico clínico en el que dictamina: manifestaciones psicóticas, esquizofrenia y reacciones depresivas de paranoia.

Finalmente la actriz, después de pasar unos días de gran inquietud, en los que el médico está más preocupado que nunca por su equilibrio mental, aparece muerta en su casa la madrugada del día cinco de agosto de 1962, a la edad de 36 años. El informe de la policía se cierra con la conclusión de "probable suicidio".

\section{Posible diagnóstico}

- Trastorno Reactivo de la Vinculación en la infancia.

- Trastorno de Personalidad Límite en la vida adulta.

En el caso que hemos relatado se podría concluir que también cumple los criterios diagnósticos del Trastorno Histriónico de la Personalidad. Sin embargo, al ser la Personalidad Límite de mayor gravedad y ser considerada por algunos autores (Cardenal, Sánchez y Ortiz-Tallo, 2007; Gradillas, 2001; Millon y Davis, 1998) como una forma maligna de personalidad histriónica nos parece más adecuado realizar éste diagnóstico.

\section{Justificación del diagnóstico}

A continuación se justifica con ejemplos el diag- 
Figura 1. Criterios Diagnósticos para el Trastorno Reactivo de Vinculación en la infancia (Manual Diagnóstico y Estadístico de los Trastornos Mentales, DSM-IV-R)

\section{Trastorno Reactivo de la Vinculación de la infancia o la niñez}

A. Relaciones sociales en la mayor parte de los contextos sumamente alteradas e inadecuadas para el nivel de desarrollo del sujeto, iniciándose antes de los 5 años de edad, y puestas de manifiesto por 1 o 2:

(1) incapacidad persistente para iniciar la mayor parte de las interacciones sociales o responder a ellas de un modo apropiado al nivel de desarrollo, manifestada por respuestas excesivamente inhibidas, hipervigilantes, o sumamente ambivalentes y contradictorias (p. ej., el niño puede responder a sus cuidadores con una mezcla de acercamiento, evitación y resistencia a ser consolado, o puede manifestar una vigilancia fría).

(2) vínculos difusos manifestados por una sociabilidad indiscriminada con acusada incapacidad para manifestar vínculos selectivos apropiados (p. ej., excesiva familiaridad con extraños o falta de selectividad en la elección de figuras de vinculación).

B. El trastorno del Criterio A no se explica exclusivamente por un retraso del desarrollo (como en el retraso mental) y no cumple criterios de trastorno generalizado del desarrollo.

C. La crianza patogénica se manifiesta al menos por una de las siguientes características:

(1) Desestimación permanente de las necesidades emocionales básicas del niño relacionadas con el bienestar, la estimulación y el afecto.

(2) Desestimación persistente de las necesidades físicas básicas del niño.

(3) Cambios repetidos de cuidadores primarios, lo que impide la formación de vínculos estables (p. ej., cambios frecuentes en los responsables de la crianza).

D. Se supone que el tipo de crianza descrita en el Criterio C es responsable del comportamiento alterado descrito en el criterio A (p. ej., las alteraciones del criterio A empezaron tras la instauración de los cuidados patogénicos que aparecen en el Criterio C).

F94.1 Tipo inhibido: si predomina el Criterio Al en la presentación clínica.

F94.2 Tipo desinhibido: si predomina el Criterio A2 en la presentación clínica.

Figura 2. Criterios Diagnósticos para el Trastorno Límite de la Personalidad (Manual Diagnóstico y Estadístico de los Trastornos Mentales, DSMIV-R)

\section{Trastorno de Personalidad Límite}

Un patrón general de inestabilidad en las relaciones interpersonales, la autoimagen y la afectividad, y una notable impulsividad, que comienza al principio de la edad adulta y se dan en diversos contextos, como lo indican cinco o más de los items siguientes:

(1) Esfuerzos frenéticos para evitar un abandono real o imaginario.

(2) Un patrón de relaciones interpersonales inestables e intensas caracterizado por la alternancia entre los extremos de idealización y devaluación.

(3) Alteración de la identidad: autoimagen o sentido de sí mismo acusada y persistentemente inestable.

(4) Impulsividad en al menos dos áreas, que es potencialmente dañina para sí mismo (p. ej. gastos, sexo, abuso de sustancias, conducción temeraria, atracones de comida.

(5) Comportamientos, intentos o amanazas suicidas recurrentes o comportamiento de automutilación.

(6) Inestabilidad afectiva debida a una notable reactividad del estado de ánimo (p. ej. episodios de intensa disforia, irritabilidad o ansiedad que suelen durar unas horas y rara vez unos días).

(7) Sentimientos crónicos de vacío.

(8) Ira inapropiada e intensa o dificultades para controlar la ira (p. ej. muestras frecuentes de mal genio, enfado constante, peleas físicas recurrentes).

(9) Ideación paranoide transitoria relacionada con el estrés o síntomas disociativos graves.

nóstico en la infancia y posteriormente el diagnóstico en la edad adulta:

\section{Trastorno de la infancia}

(Trastorno reactivo de la vinculación. Tipo Desinhibido)

- La niña Norma Jean tiene relaciones sociales en la infancia inadecuadas con figuras de referen- cia extremas que plantean pautas educativas diametralmente opuestas. Falta de selectividad en la elección de figuras de apego en la edad. (Criterio A 2 del DSM-IV-R).

- No parece que pudiera haber ningún problema de retraso mental o del desarrollo tal como reflejan los comentarios en la ficha de uno de los Colegios en los que estuvo. (Criterio B del DSM-IV-R). 
- En su infancia cambia al menos, 5 veces, de cuidadores y de hogar. En primer lugar con padres adoptivos, posteriormente su madre biológica, amiga de la madre, centros de acogida y varios padres adoptivos. (Criterios $\mathrm{B}$ y $\mathrm{C}$ del DSM-IV-R).

- Es muy probable que la influencia de la crianza que recibió haya influido notablemente en la inestabilidad afectiva en su época adulta (Criterio D del DSM-IV-R).

\section{Trastorno en la edad adulta}

(Trastorno de Personalidad Límite)

- Marilyn realiza a lo largo de su vida adulta enormes esfuerzos por evitar el abandono imaginario o real de sus constantes amantes, de sus cuatro maridos y de sus íntimas amigas. En algunos momentos llevando a cabo algún intento de suicidio ante el temor del abandono del marido. (Criterios I y III del DSM-IV-R).

- Sus fluctuaciones de ánimo llevan a tener que suspender y reanudar constantemente los ensayos por su incapacidad para cierta estabilidad. (Criterio II del DSM-IV-R).

- Relata desde muy joven tener sentimientos crónicos de vacío. (Criterio 6 del DSM-IV-R).

- Se relaciona de forma muy intensa con distintos personajes masculinos y femeninos a lo largo de su vida. Sus relaciones son de gran dependencia emocional y en muchos de los casos se mueve entre la necesidad de dependencia y la necesidad de rebelarse. (Criterios 2 y 3 del DSM-IV-R).

- Toma drogas diariamente. (Criterio 4 del DSMIV-R).

- Relatan que se autolesiona y tiene varios intentos de suicidio a lo largo de su vida adulta. (Criterio 5 del DSM-IV-R).

- Estando hospitalizada refieren que tenía lo que los médicos denominaron "ataques de histeria". Estos ataques le hacen tirar los muebles de la habitación y proferir gritos a todo el que le rodea. (Criterio 8 del DSM-IV-R).

- El psiquiatra que la trata durante el último año ante la diversidad de síntomas que presenta, realiza un diagnóstico clínico en el que dictamina: manifestaciones psicóticas, esquizofrenia y reacciones depresivas de paranoia. (Criterio 9 del DSM-IV-R).

\section{Características clínicas de la personalidad límite}

Los Trastornos de Personalidad, en general, hacen referencia a formas patológicas habituales de comportarse. El trastorno de personalidad límite, del que se trata en este artículo, es una de las personalidades que mayores dificultades presenta para su identificación y comprensión.

Su dificultad proviene de la diversidad y la complejidad de los síntomas que presenta. Esto supone que la forma de aparición en la clínica sea también muy variada y compleja. La mayoría de estas personas acuden a consulta a pedir ayuda de forma urgente y un tanto dramática. Cualquier hecho puede haber provocado en la persona un desequilibrio importante: el temor a una ruptura sentimental, un cambio de planes o una simple contradicción a sus deseos puede provocar alteraciones diversas, periodos de abatimiento intensos, cambios de humor o incluso un intento de suicidio. En algunos casos es la familia quien desesperada pide orientación por el comportamiento extraño y peculiar que identifican como caprichoso, con llamadas de atención en casa, con explosiones de ira, o cambios de humor que hacen difícil la convivencia.

\section{Variabilidad e intensidad de los estados de ánimo}

Una de las principales características de esta personalidad es la intensidad y la variabilidad de sus afectos y de sus estados de ánimo. Muestran marcados cambios en su estado de ánimo que van desde la normalidad a la depresión, a la irritabilidad o a la excitación. Estos cambios no tienen que ir asociados a variaciones en su ambiente exterior, sino que pueden aparecer sin motivos aparentes.

Las fluctuaciones no son constantes, sino que con frecuencia cada personalidad límite tiene su estado de ánimo dominante (depresivo, irascible, excitado) y periódicamente da paso de forma repentina a 
periodos o momentos variados y de gran intensidad. Las fluctuaciones de su estado de ánimo y de su equilibrio emocional son tan extraordinarias que afecta a toda su vida. Algunos autores consideran que el término apropiado para esta característica es el de labilidad, cambios rápidos y súbitos del estado de ánimo.

Es fácil que de estos cambios surjan problemas en su comportamiento lo que daría lugar a la característica siguiente.

\section{Comportamientos impulsivos}

Realizan de forma impulsiva acciones, que más tarde pueden llegar a reconocer como irracionales o contraproducentes. Es como si tuvieran cierta incapacidad para resistir cualquier tipo de impulsos. Así es frecuente que se den atracones de comida, que derrochen dinero, que compren compulsivamente, que realicen juegos de azar, que abusen de sustancias o que conduzcan temerariamente. Estos comportamientos que podríamos denominar también irreflexivos suelen conllevar peligro para ellos y para sus familiares.

Muy ligada a esta forma impulsiva de comportarse estaría la siguiente característica que, por su importancia o por su gravedad se comenta aparte.

\section{Autoagresividad}

Los comportamientos autodestructivos en el que se destaca el comportamiento suicida son frecuentes en estas personas. Así pueden realizar veladas amenazas de suicidio, expresiones verbales acerca de esa posibilidad o claras amenazas a los familiares si no consigue algo que tiene interés. Precisamente por su impulsividad y por su labilidad de ánimo estas amenazas hay que considerarlas porque frecuentemente llevan a cabo el intento en momentos impulsivos y de fluctuación.

Los comportamientos autolesivos, como cortes, quemaduras, golpes, arañazos se observan también frecuente y reiteradamente en su vida.

Otra forma de comportamiento autodestructivo de estas personas es dejar un determinado objetivo justo cuando están a punto de conseguirlo, ejemplos de ello sería abandonar el curso escolar en los exámenes finales, romper una relación cuando se ha consolidado, etc.

\section{Preocupación excesiva por el abandono y la soledad}

Estas personas son muy sensibles a la pérdida de una relación interpersonal importante. Apenas se percatan de la inminente ruptura, real o supuesta, de una relación significativa se producen cambios profundos en la afectividad, en el comportamiento y en los pensamientos.

La intolerancia al abandono es tal que hasta la separación transitoria (un viaje de un familiar o de una persona significativa) le lleva a sentir un pánico intenso y unas reacciones coléricas claramente inadecuadas.

Esta preocupación le puede llevar a un descontrol y a realizar esfuerzos impetuosos para conseguir que no se realice, dando así lugar a reacciones autolesivas o a comportamientos suicidas que se han comentado anteriormente.

\section{Alteraciones de la identidad}

Las fluctuaciones afectan a la imagen o al sentido de uno mismo. La propia imagen tiene fluctuaciones, apareciendo así nuevos gustos, objetivos, valores, ideales, vocación profesional o identidad sexual.

Tienen dificultades considerables para mantener un sentido estable de quiénes son, cambiando rápidamente la imagen del sí mismo. También poseen representaciones del sí mismo contradictorias, reflejándose así la falta de cohesión interna.

A veces, la alteración de la identidad es tan intensa que tienen la sensación como de no existir.

\section{Relaciones interpersonales intensas e inestables}

Las relaciones interpersonales dan la sensación de oscilar entre la superficialidad, la dependencia y la manipulación. Al ser intensas e inestables son turbu- 
lentas y tienen propensión a cambiar súbita y drásticamente de actitud hacia las personas. Así, de ensalzar las cualidades de alguien pasan a su más completa desvalorización.

Esta característica puede tener que ver con el temor al abandono y con el deseo excesivo de protección. Si piensa que la persona allegada, madre, padre, terapeuta, etc... no le está prestando suficiente atención puede cambiar bruscamente su actitud hacia esa relación.

\section{Actitud muy exigente}

Todas las características de dependencia y las fluctuaciones comentadas le llevan a tener una actitud muy exigente con las personas cercanas. La sensación que produce en los demás es que se trata de una actitud y un comportamiento ante la vida caprichoso. Esto suscita con frecuencia en las personas cercanas reacciones confusas y conflictivas, como no comprender al paciente, estar harto de sus manipulaciones y exigencias, considerar sus exigencias caprichos continuos, lo que lleva con facilidad a reacciones desesperadas de los allegados creándose así un círculo vicioso en el que la persona aumenta sus exigencias y reacciona más impulsivamente.

\section{Episodios psicóticos breves}

Suele haber rupturas breves con la realidad que, sin embargo, ellos mismos, más tarde identifican como desviado o extraño. Así pueden tener ideas delirantes, alucinaciones, períodos de comportamiento extraño o estados de ánimo muy extremos.

La aparición de los episodios psicóticos pueden estar relacionados con periodos de estrés importantes tales como viajes, cambios en su vida, etc...; sin embargo preservan lo esencial del juicio de realidad. Así pueden describir minuciosamente un delirio de persecución o explicar una alucinación auditiva, pero teniendo cierta capacidad de crítica acerca de la certeza o no de su pensamiento o de su percepción. Los síntomas psicóticos frecuentemente son de contenido condenatorio por haber realizado acciones reprobables (que pueden ser ciertas) o de culpa por su comportamiento bizarro.

\section{Gran variedad de síntomas}

El paciente con características límites puede aparecer de un día para otro con síntomas diversos y que podrían hacer sospechar la existencia de otra patología o la confusión del diagnóstico. Así expresa quejas somáticas que pueden hacer sospechar enfermedades graves de diversa índole (somatizaciones); aparecen síntomas que afectan las funciones motoras o sensoriales y que sugieren algún trastorno neurológico (síntomas de conversión); actitudes hipocondríacas; incapacidad para recordar información personal importante (amnesia disociativa); estados de ánimo depresivos, irascibles y excitados que sugieren un trastorno del estado del ánimo y; crisis y estados de ansiedad que acompañan a la personalidad límite en su difícil peregrinaje. Por último parece existir una fuerte asociación en la sociedad actual entre la personalidad límite y el abuso de sustancias, así se encuentra frecuentemente pacientes que abusan de diferentes sustancias, entre las que se encontrarían el alcohol, la cocaína y el crack.

\section{Diagnóstico diferencial}

Esta variedad en su presentación requiere por parte del clínico un profundo conocimiento del patrón de comportamiento para poder distinguirlo de otras alteraciones psicopatológicas que comparten los mismos síntomas y que en un primer acercamiento podrían ser confundidas.

Su complejidad incluso afecta al mismo término empleado para designar esta personalidad. El término límite se comienza a utilizar como entidad diagnóstica oficial a partir del DSM-III (1980). Aún estando los diversos profesionales que componían la comisión de acuerdo con los criterios diagnósticos que integraban este patrón de comportamiento, no ocurría lo mismo con el término elegido para designar esta forma de ser patológica. Las diferencias de opiniones acerca del término más correcto han con- 
formado la historia del término aplicado al Trastorno de Personalidad.

Los argumentos que dan los autores que no creen correcta la denominación de Límite son variados. Es una designación vaga y general para representar lo que es una constelación de rasgos sindrómicamente distintiva. No tiene ningún significado clínico ni descriptivo. En el sentido puramente linguístico, el término sólo transmite clara y útilmente un nivel moderado de gravedad o un grado intermedio del funcionamiento de la personalidad. El término ha sido empleado para designar la intensidad de un fenómeno psicopatológico como la capacidad intelectiva (p. ej. La deficiencia mental leve que se confunde con los niveles más bajos de la normalidad). Por último, también ha sido empleado para designar cuadros clínicos con manifestaciones entre dos grupos de trastornos (p. ej. neurosis y psicosis).

La Clasificación de la OMS (CIE) denomina, sin embargo, quizá de forma más acertada, al mismo patrón de comportamiento Trastorno de inestabilidad emocional, considerando o subdividiéndolo en dos tipos el tipo impulsivo y el tipo límite.

Millon (1998), autor de reconocido prestigio en el estudio de los Trastornos de Personalidad propone una serie de términos que considera más adecuados: personalidad lábil; trastorno ambivalente de la personalidad, trastorno impulsivo de la personalidad o trastorno inestable de la personalidad, considerando el término límite inapropiado, pobre de contenido, absurdo y dado a malinterpretaciones.

Realizar un diagnóstico diferencial entre los TP Límite y otros trastornos del eje I y II no siempre es fácil. El motivo principal es que frecuentemente la personalidad límite se acompaña o está asociada a otros trastornos bien del eje I o bien de otros trastornos de la personalidad. Por otro lado, y a pesar que en los sistemas de clasificación DSM-IV y CIE-10 han intentado reducir la superposición entre diagnósticos, las investigaciones más actuales y las futuras se encaminan a tratar de realizar un acercamiento dimensional de las alteraciones, identificando las dimensiones básicas que subyacen a los diversos trastornos de personalidad. En este sentido, algunos estudios ya apuntan la identificación de algunos factores que son únicas o exclusivas de determinados trastornos de personalidad (Livesley \& Schroeder, 1991).

En concreto, para el TP Límite se ha encontrado que el factor de inestabilidad o desorganización es característico exclusivamente de esta personalidad. Mientras que otras de sus características clínicas, como la forma de relacionarse socialmente o la conducta autolesiva, son compartidas con otros diagnósticos.

Aún así, el solapamiento entre los diversos trastornos de personalidad es considerable y está bien documentada. Los estudios al respecto, muestran que el TP Límite en su estado puro y sin otros diagnósticos compartidos aparece sólo en el 3-10\% de los casos. El motivo, para algunos autores es un problema relacionado con la continuidad entre los distintos trastornos. Las fronteras entre ellos no son tajantes ni precisas, sino que comparten en mayor o menor grado características similares. De nuevo tendríamos que volver a hablar de dimensiones continuas y no de clasificaciones categoriales. Una serie de estudios sugieren que el límite no es una categoría discreta sino que más bien se definiría como una forma severa de neuroticismo con contribuciones menores de otras dimensiones de personalidad.

Veamos a continuación, aquellos trastornos que por su similitud o por su aparición conjunta están más estrechamente ligados al que nos ocupa. Comenzaremos distinguiéndolo de un trastorno del eje I para posteriormente tratar de establecer distinciones con otros trastornos de la personalidad:

\section{Trastornos del estado del ánimo Bipolar}

Algunos investigadores se plantean la posibilidad de que el trastorno límite de la personalidad sea una variante de los trastornos bipolares, dada la estrecha relación que presentan ambos tipos de pacientes. Cuando aparecen los síntomas psicóticos es también difícil hacer la distinción observando únicamente los síntomas clínicos.

Sin embargo, la principal diferencia reside en la historia del desarrollo de la alteración: Los Trastornos del estado del ánimo con síntomas psicóticos suelen tener un inicio rápido y además, aunque en su historia hayan tenido dificultades en las rela- 
ciones interpersonales la mayoría han mantenido un equilibrio previo, al menos durante bastante tiempo seguido. El límite ha tenido una historia de funcionamiento inferior y menos adaptativa. En el caso descrito en este artículo se puede comprobar.

Otra diferencia tiene lugar cuando se observan los desencadenantes de las alteraciones. La alteración del límite parece reflejar defectos internos, no dependiendo tanto sus cambios de estresores externos, el bipolar, al menos, en parte suele estar influido por estresores externos o ambientales.

Por último el paciente bipolar siente el trastorno del estado del ánimo como egodistónico, discordante con su forma de ser y como algo ajeno. El límite soporta con relativa comodidad determinados rasgos patológicos que parecen estar más arraigados en lo más interno de su personalidad.

\section{Trastorno por estrés postraumático}

Kroll (1993) afirma que las personas que presentan un TP Límite y un Trastorno por Estrés Postraumático presentan en primer término un trastorno de la conciencia. Ambos tipos de personas son incapaces de desconectarse de una conciencia que se ha convertido en su propio enemigo, compuesta por recuerdos reales de sucesos traumáticos, recuerdos distorsionados y fragmentados, sensaciones físicas desagradables, comentarios negativos sobre sí mismos, elaboraciones fantaseadas y temidas; todo ello probablemente consecuencia de una infancia con abusos. Siendo su estado un intento por acabar o por modificar su propia conciencia.

En el caso descrito la historia de posibles abusos en la infancia podría incluir este diagnóstico. La relación entre abusos en la infancia y posterior trastorno límite de la personalidad es objeto de investigaciones.

\section{Trastorno histriónico de la personalidad}

Es quizá éste el trastorno de personalidad que más aparece asociado o que más se puede confundir con el límite. El compartir ambos el criterio de la inestabilidad emocional hace que en la clínica su aspecto pueda confundirse. Diversos autores conciben el Trastorno Límite como un nivel de patología más avanzado que interrelaciona con otros estilos de personalidad menos graves como el histriónico. Podría incluso decirse, en ocasiones, que el límite es la forma "maligna" del histriónico.

Ambos dan muestras de labilidad emocional asociada a manipulaciones interpersonales y búsqueda de atención. Una de las diferencias es que los límites tienen arranques de cólera y repetidos sentimientos de vacío y soledad que no caracterizan a la personalidad histriónica. La intensidad del afecto y del estado de ánimo son en ambas muy exageradas, la diferencia estaría que en el trastorno histriónico el afecto parece más expresado que sentido, más superficial; mientras el límite experimenta realmente un afecto y un estado de ánimo intenso y no se observa tanto la apariencia de superficialidad.

En el caso expuesto, se podría decir que incluye el diagnóstico de trastorno de personalidad histriónico, pero al ser más grave y comprender el trastorno límite a éste el diagnóstico creemos más correcto sería éste último.

\section{Trastorno antisocial de la personalidad}

En este caso, ambas personalidades pueden ser graves y ambas manipuladoras en sus comportamientos y actitudes. Sin embargo, la personalidad antisocial manipula con la finalidad de aprovecharse de los demás y obtener poder, a diferencia del límite que en sus manipulaciones lo que trata de hacer es descargar sus intensas emociones y atraer la atención y el cuidado de los demás.

\section{Trastorno de personalidad esquizotípico}

Para realizar la distinción entre ambos diagnósticos, límite y esquizotípico, convendría recordar uno de los criterios establecidos por el DSM-IV. Este es, la ideación paranoide transitoria relacionada con el estrés o síntomas disociativos graves.

Esta inclusión realizada en la último revisión de la APA acerca considerablemente a los dos trastornos mencionados. 
Sin embargo, la distinción fundamental se encontraría en que en la personalidad límite las experiencias psicóticas y disociativas son transitorias y breves, sin embargo, en la personalidad esquizotípica son síntomas estables y crónicos.

\section{Hipótesis explicativas}

\section{Factores de predisposición}

\section{a) Factores socioculturales}

Los valores y las prácticas de la sociedad actual parecen ser más inconsistentes que las de épocas anteriores. Según Millon y Davis $(1998,2001)$ bajo el impacto de la inmigración, de la movilidad, de la tecnología y de la comunicación de masas, se ha producido una erosión de los valores y las costumbres tradicionales. En este marco, a los niños les es difícil tener una serie de prácticas y creencias coherentes y estables, enfrentándose en pocos años a cambios de valores y al cuestionamiento de normas que presidían su vida poco antes. Esto puede provocar que determinados niños se desconcierten ante la falta de una visión coherente y estable de la vida y sean incapaces de encontrar una estabilidad en sus relaciones y en su idea del mundo.

Desde este punto de vista, el niño con propensión a un trastorno límite estaría más respaldado en un entorno amplio, familia extensa, barrio, profesores cercanos, etc... que ayudarían a tener diferentes modelos en los que guiarse y sentirse más seguro. Sin embargo, hoy en día son frecuentes las familias monoparentales, las relaciones con los maestros más distantes; lo que podría colaborar en la sensación de vacío, de soledad y de inconsistencia del futuro límite.

\section{b) Factores de la Historia familiar}

Centrándonos en influencias u orígenes psicológicos, investigaciones al respecto, encuentran que la historia familiar de los TP Límites suelen incluir fronteras familiares poco claras, relaciones caprichosas entre los miembros de la familia y posibilidad de abusos infantiles.
En relación a este último aspecto, la mayor incidencia de abusos infantiles en los pacientes límites ha llevado en las dos últimas décadas a que se realicen estudios centrándose en ello. Sin embargo, los resultados de los estudios empíricos acerca de la existencia de abusos en la infancia de las personas con TP Límite, son complejos y en cierto modo, ambiguos. Aunque parece haberse encontrado con relativa frecuencia el abuso sexual en la infancia de estos pacientes, no todos han sufrido esta experiencia y; por otro lado, no todas las experiencias de abusos sexuales en la infancia suponen la aparición de un TP Límite.

Se podría decir que los abusos, en el sentido amplio del término, tanto abusos sexuales, abusos físicos (castigos, violencia doméstica) como los abusos psicológicos (negligencia de los padres, abandono, etc...) pueden predisponer o ser uno de los factores de riesgo de importancia para la formación de un límite.

Pero con la dificultad que siempre supone establecer causas directas y claras en la formación o aparición de cualquier trastorno psicopatológico o trastorno de la personalidad, no se puede establecer como algo determinante el abuso en la historia familiar, ni es un aspecto imprescindible para la aparición de este trastorno.

\section{c) Factores de la historia personal}

En la historia infantil de la personalidad límite se suele encontrar que son niños con un grado de imprevisibilidad comportamental y un estado de ánimo muy variable. Muchos suelen haber tenido mal genio, han sido niños irritables, inquietos y propensos a grandes rabietas. Algunos pueden haber sido extremadamente dependientes y otros muy miedosos, con fobias y con comportamientos hipocondríacos.

Algunos autores sugieren que las raíces de este trastorno se encuentran en un Trastorno por déficit de atención con hiperactividad en la infancia.

En la edad adolescente se pueden caracterizar por tener sentimientos de apatía y aburrimiento y salvo por las gratificaciones inmediatas, no encontrar satisfacción ni sentido a su vida. Incapaces, por lo tanto, de encontrar un sentido a su vida, no planifi- 
can su vida ni se comprometen a ir cumpliendo objetivos. Incapaces, también, de seguir una disciplina y cumplir con las demandas de la vida académica, carecen de un grupo coherente de valores a través de los cuales canalizar sus energías y junto con su autoimagen previa negativa y su inestabilidad buscan reafirmarse a través de comportamientos autodestructivos y variables.

\section{Factores de mantenimiento}

El propio estilo de comportamiento del patrón de personalidad límite facilita su proceso de autoperpetuación.

Su necesidad de dependencia y, sobre todo, su intenso temor al abandono hace que tengan comportamientos peculiares para evitar las separaciones.
Pueden poner todo su empeño en utilizar la tristeza, el abatimiento y la depresión para evitar una separación y si de esta forma no lo consiguen pasar a otro estilo de afrontamiento inverso se vuelven frívolos, irascibles exigentes o agresivos. Sus objetivos son siempre los mismos evitar el supuesto abandono, sin embargo si no lo consiguen y cambia constantemente de estrategia en su desesperación, puede significar el mantenimiento de una de sus principales características: la variabilidad.

Al necesitar liberar la tensión que les produce su alta emocionalidad se quejan exageradamente, expresan su ira contra sus cuidadores manteniendo en jaque a todos los que le rodean y consiguiendo de esta forma el efecto que tanto temen el rechazo y el alejamiento de los demás. Esto también hará, por lo tanto, que se agudicen sus síntomas y se autoperpetúe su estilo.

\section{Extended Summary}

The aim of this study is to help the reader in understanding a complex and severe personality disorder such as borderline personality disorder. In this case study it is also possible to come near a diagnosis of a childhood disorder, one which is similarly complex and infrequent, such as Reactive Attachment Disorder in childhood.

The data that will be set out has been compiled through the reading of documents, such as several biographies written by different authors of a popular film star (e.g. Guiles, 1985; Leaming, 2007; Pepitone \& Stadiem, 1979; Reig, 2005; Schneider, 2008; Spoto 2000). Because of the way that the information has been recovered, this case study is a retrospective one, with the limitations that this implies.

\section{Presentation of the case}

It is the case of a woman who was married on four occasions, who had no children and who worked as an actress. She was born in June 1926 and deceased on August the $5^{\text {th }}, 1962$.

Family history. Her mother is described as a woman of an unstable and insecure personality. When the woman gets pregnant with this baby, whom she will call Norma J., she does not know for sure who the father might be. After birth, the baby is left under the care of a foster family that has several foster children for whom they receive money from the state.

During the baby's first years, her maternal grandmother passes away and it appears that she had a manic-depressive psychosis diagnosis. For the subject's mother and for herself, the fear of belonging to a family with a history of mental disorders that could be inherited remains forever.

During these first seven years of existence, although there is no proof of abuse, Norma lives a period where the signs of psychological and affective tension are constant due to her partly unknown origin and the fact that her biological mother could show up and disappear at equal speed.

Her upbringing with her foster parents has been defined as "terribly strict". The church was the center of her life, cinema and actors circles were considered to be sinful.

When she is seven, her mother takes her to live with her and some actors that share the home with them. For the little girl it means going to the exact opposite environment from the one she had experienced for her first years of life. The huge difference in the way she was been brought up now and how she had been during her first childhood creates a 
considerable confusion in the little girl about what appropriate behavior would be, and knowing what guidelines to follow.

When she turns eight, her maternal great grandfather hangs himself from a rafter. This event puts her mother, who was scared of the family curse, in such a state of depression that she has to be admitted in mental hospital.

Meanwhile, the little girl is been taken care of by her mother's friend, Grace, who leads her through a whole new behavioral world. Seduction, mimicking artists and flashy outfits that were quite inappropriate for her age, are the general rules for this new period of her life. Thus, her childhood is defined as a constant chain of contradictions.

From 9 to 11 she goes back to an orphanage and she sinks into a darker mood. It would seem that there were episodes of sexual abuse from the age of 11 until she was 13.

She starts getting her period before most of the girls her age, this allows her to attract the boys' attention and develop an enormous capacity of seduction and attraction that she will use constantly along her life.

She marries when she is 15 ; she has several lovers at the time and goes through periods of great depression.

She starts taking drugs, tranquillizers, and she will be addicted to them until the end of her life. When she is 20 , she starts working as an actress, changes producers and has intense and unstable romantic relationships with a number of producers and actors. It is at that time that they decide to change her name into a more appropriate one for show business, Marilyn Monroe.

She marries on three further occasions and several relationships with women are attributed to her. On 1949 , a new phase of her career starts where everybody is impressed by her beauty and her capacity to show feelings and emotions. However, her cast colleagues complain of having to work with Marilyn. She is unstable, fails to meet her obligations and is very moody. She tries to commit suicide and is admitted to hospital several times.

She goes into deep depression; she expresses a great self-destruction feeling and a huge sense of emptiness. On occasions, she confuses reality and fiction. On 1961 she enters another mayor crisis period, the psychiatrist that has been treating her along the past year finds out she is taking drugs on a daily basis and, surprised by the number of symptoms she shows, states on his clinical diagnosis: hysteria, psychotic symptoms, schizophrenia and paranoiac depressive reactions.

Finally, after spending some days in a very uneasy state while her doctor is more worried than ever about her mental health, the actress is found dead in her home on August the $5^{\text {th }}, 1962$. She was then 36 years old. The police file is closed concluding a "probable suicide".

Probable diagnosis. A Reactive Attachment Disorder in childhood and Borderline Personality Disorder in her adult life.

It could also be concluded that she reaches the criteria for a histrionic personality disorder. However, as the borderline personality is considered to be a more severe disorder and somehow a malign form of histrionic personality, we believe the above diagnosis to be more appropriate.

\section{Diagnosis justification}

\section{Reactive Attachment Disorder. The Uninhibited Type}

- The subject has inadequate social relationships during her childhood, with extreme reference figures that educate her with opposite guidelines. Lack of selectivity when choosing attachment figures in early age. (A 2 criterio on the DSM-IV-R)

- It does not seem that there could be a problem of mental or development delay. (B criterion on the DSM-IV-R)

- She changes home and city for at least five times along her childhood. She lives with foster parents, with her mother, her mother's friend, in children institutions and several other foster parents. (B and C criteria on the DSM-IV-R).

- It is very probable that her upbringing has had a high influence on the affective instability she suffered throughout her adult life. (D criterion on the DSM-IV-R). 


\section{Borderline Personality Disorder}

- Marilyn makes enormous efforts throughout her adult life in order not to loose, either in reality or in her imagination, her continuous lovers, her four husbands and her best female friends. On several occasions she committed an attempted suicide out of fear of loosing a husband. (I and III criteria on the DSM-IV-R).

- Rehearsals are continuously interrupted because of her moodiness and her incapacity of certain stability. (II criterion on the DSM-IV-R).

- She expresses having cronical feelings of emptiness from a very early age. (Criterion 6 on the DSM-IV-R).

- Along her life, she relates to different people, both male and female, in a very intense manner. Her relationships are very dependent on an emotional level and in a lot of cases she shifts from her dependency to the need of rebellion. (Criteria 2 and 3 on the DSM-IV-R).

- She takes drugs on a daily basis. (Criterion 4 on the DSM-IV-R).

- She harms herself and commits several attempted suicides along her adult life. (Criterion 5 on the DSM-IV-R).

- While being in hospital there are references to what doctors call "hysteria crises". These crises make her throw the furniture in her room and shout at everybody around her. (Criterion 8 on the DSM-IV-R).

- The psychiatrist that treats her throughout the last year of her life, in accordance with the number of symptoms she shows, states on his clinical diagnosis: hysteria, psychotic symptoms, schizophrenia and paranoiac depressive reactions. (Criterion 9 on the DSM-IV-R).

\section{Explaining hypothesis}

\section{Tendency factors}

\section{a) Sociocultural factors:}

The behavior and values of society at the time seem to be more inconsistent than in previous years.
This can provoke certain children to be confused over the lack of a clear and stable vision of life and thus, to be incapable of finding stability in their relationships and their idea of the world.

From this point of view, a child with a borderline disorder tendency would be better taken care of in an expanded context, with a large family, a neighborhood, teachers that are close to students, etcetera, this would help them by providing different role models and making them feel more secure. However, nowadays single-parent homes are frequent, the relation with the teachers is more distant; this could increase the feelings of emptiness, loneliness and inconsistency of the future borderline personality.

\section{b) Family history factors}

According to studies on the matter, family history of people with a borderline personality disorder has a tendency of including unclear family boundaries, unstable relationships among family members and the probability of child abuse.

We could say that abuses, in the wider sense of the term (sexual abuse, physical abuse such as punishment or domestic violence, psychological abuse such as uncaring parents, abandonment, etcetera), can be an important risk factor for those who have the tendency to develop a borderline personality.

However, considering the difficulty in establishing direct and clear causes for the development of any personality disorder, the abuse in the family history can not be considered a determining factor, neither it is a required feature in this kind of disorder.

\section{c) Personal history Factors}

In the childhood history of borderline personality cases it is often the case that we find children with high behavioral unpredictability and moodiness. A large number of them had bad temper, were irritable, restless and had a tendency to big temperamental outbursts. Some of them might have been very dependent, others very fearful, phobic and had hypochondriac behavior. 
During their adolescence they can experience feelings of listlessness and boredom, not finding any reward or meaning in their lives except for instant or immediate gratification.

\section{Maintenance Factors}

It is the very behavioral style of those with standard borderline personality that contributes to the process of self-perpetuation that they suffer.

Their need for dependency and, even more so, their intense fear of being abandoned, make them behave peculiarly in order to avoid separations. Because they need to liberate the tension created by their emotional ordeal, they complain constantly, they express their anger against their care-givers, keeping everybody on edge in such a way that it results in what they fear the most, people reject and leave them. This will lead to more acute symptoms and thus, to the perpetuation of the type.

\section{Referencias}

American Psychiatric Association (APA) (1980). Diagnostical and Statistical Manual of mental disorders, III (DSM-III). Washington, D.C.: American Psychiatric Association (APA). (1952, DSM-I; 1968, DSM-II; 1987, DSM-III-R; 1994, DSM-IV; 2000, DSM-IV-TR).

Cardenal, V., Sánchez, P. y Ortiz-Tallo, M. (2007). Los trastornos de personalidad según el modelo de Millon: una propuesta integradora. Clínica y Salud, 18, 3.

Gradillas, V. (2001). Trastornos de la personalidad en la práctica médica. Madrid: Masson Elssevier.

Guiles, F.L. (1985). Legend: The Life and Death of Marilyn Monroe. New York: Amazom.

Kroll, J. (1993). PTSD/borderlines in therapy: Finding the balance. New York: W. W. Norton.

Leaming, B. (2007). Marilyn Monroe. New York: Amazom.

Livesley, W.J. y Schroeder, M. (1991). Dimensions of personality disorders. The DSM-III-R cluster B diagnosis. Journal of Nervous and Mental Disease, 179, 320-328.

Millon, Th. y Davis, R.D. (1998). Trastornos de la personalidad más allá del DSM-IV. Barcelona: Masson.

Millon, Th. y Davis, R.D (2001). Trastornos de la Personalidad en la vida moderna. Masson: Barcelona.

Ortiz-Tallo, M. y Cardenal, V. (2004). El apasionante mundo de la personalidad. Archidona (Málaga): Ediciones Aljibe.

Pepitone, L. y Stadiem, W. (1979). Marilyn Monroe Confidential: An intimate personal account. New York: Simon and Schuster.

Reig, R. (2005). Autobiografía de Marilyn Monroe. Madrid: Ediciones Lengua de Trapo.

Schneider, M. (2008). Ultimas sesiones con Marilyn. Madrid: Alfaguara

Spoto, D. (2000). Marilyn Monroe. Madrid: Anagrama.

Manuscrito recibido: 09/02/2010

Revisión recibida: 22/02/2010

Manuscrito aceptado: 08/03/2010 